\title{
Berberine inhibits cardiac remodeling of heart failure after myocardial infarction by reducing myocardial cell apoptosis in rats
}

\author{
YING LIAO*, KAIHONG CHEN* , XINGMO DONG, WEIGUO LI, GANYANG LI, \\ GUOYONG HUANG, WEI SONG, LILING CHEN and YONG FANG \\ Department of Cardiology, The Affiliated Longyan First Hospital of \\ Fujian Medical University, Longyan, Fujian 364000, P.R. China
}

Received January 5, 2018; Accepted June 18, 2018

DOI: $10.3892 / \mathrm{etm} .2018 .6438$

\begin{abstract}
The effects of berberine on cardiac function of heart failure after myocardial infarction and its possible mechanism were investigated. The anterior descending branches of 50 female Wistar rats were ligatured to establish the model of heart failure after myocardial infarction. At 4 weeks after successful modeling, the rats were randomly divided into two groups receiving 4-week gavage with saline (Sal group) and berberine (Ber group), while the sham-operation group (Sham group) was set up. After 4 weeks, the hemodynamics and serum BNP in rats were measured. The hearts of rats were taken to detect the degree of myocardial fibrosis. The myocardial cell apoptosis was detected. The expressions and changes in myocardial apoptosis-related proteins, including Bcl-2, Bax and caspase-3, were detected. The expression and changes in GRP78, CHOP and caspase-12 in myocardial tissue were detected. The results showed that Berberine improved the cardiac function of rats after myocardial infarction. After myocardial infarction, myocardial fibrosis and apoptosis were observed around the infarction area, berberine improved the myocardial fibrosis and reduced cell apoptosis. Furthermore, berberine alleviated endoplasmic reticulum stress (ERS) after myocardial infarction. In conclusion, Berberine can inhibit the myocardium cell apoptosis of heart failure after myocardial infarction, and its mechanism may be realized by affecting the ERS in myocardial tissue of heart failure after myocardial infarction and CHOP and caspase-12 apoptotic signaling
\end{abstract}

Correspondence to: Dr Yong Fang, Department of Cardiology, The Affiliated Longyan First Hospital of Fujian Medical University, 105 Jiuyi North Road, Longyan, Fujian 364000, P.R. China

E-mail: wingjays@163.com

${ }^{*}$ Contributed equally

Key words: berberine, myocardial infarction, fibrosis, apoptosis, endoplasmic reticulum stress pathway, upregulating Bcl-2/Bax expression and downregulating caspase- 3 expression, thus inhibiting the cardiac remodeling and protecting the cardiac function.

\section{Introduction}

Heart failure refers to a group of clinical syndromes caused by ventricular filling and/or impaired ejection capacity due to various cardiac structures and/or functional diseases, which is the end stage of various cardiovascular diseases (1). At present, scholars worldwide have agreed that cardiac remodeling is the root cause of the development and progression of heart failure $(2,3)$. Thus, inhibition of cardiac remodeling can improve cardiac function and long-term prognosis of patients with heart failure. Under the guidance of this theory, the clinical treatment mode of heart failure has also been transformed from the simple improvement of hemodynamics to the treatment that aims to improve the cardiac remodeling $(4,5)$, such as the inhibition of renin-angiotensin-aldosterone system (RAAS) and sympathetic nervous system. At present, the clinical drugs used to inhibit RAAS and sympathetic nervous system mainly include the $\beta$-receptor blocker, angiotensin converting enzyme inhibitor, angiotensin receptor inhibitor and aldosterone receptor antagonist. The wide application of these drugs helps significantly decrease the fatality rate and hospitalization rate of heart failure. But the morbidity and mortality of heart failure are still high, which are still the main causes of disability and death of cardiovascular diseases, seriously threatening human health $(6,7)$. Therefore, research on new anti-heart failure drugs is still in the ascendant.

The fundamental feature of heart failure is cardiac remodeling, which is the change in myocardial structure, function and phenotype due to a series of complex molecular and cellular mechanisms, manifested as pathological hypertrophy of myocardial cells, myocardial cell apoptosis, and excessive fibrosis or increased degradation of extracellular matrix (8-10). Myocardial cell apoptosis is the main reason for the continuous loss of myocardial contraction units in the development and progression of heart failure, which is related to the progressive decrease of cardiac function, and involved in the basic pathological and physiological processes of heart failure. Therefore, 
the inhibition of myocardial cell apoptosis has important clinical significance for the reduction of cardiac remodeling and improvement of cardiac function. Recent studies have shown that endoplasmic reticulum stress (ERS) is associated with heart failure, and ERS-mediated myocardial cell apoptosis is an important aspect of cardiac remodeling $(11,12)$, thus inhibiting the ERS-mediated myocardial cell apoptosis is of great importance for improvement of cardiac function.

Berberine is the main active ingredient of traditional Chinese medicine, Coptis chinensis, mainly used clinically in the treatment of bacterial infection of gastrointestinal tract. The latest study has found that berberine has not only a good anti-dysentery activity, but also a variety of cardiovascular pharmacological activity, such as antiarrhythmic effect, antiheart failure, antihypertensive effect and regulation of blood lipids (13-16). Clinical studies have confirmed that berberine has the function to enhance myocardial contractility and improve left ventricular function (17). Berberine increasingly shows a certain function in the treatment of cardiovascular disease, especially heart failure, but its mechanism of antiheart failure remains unclear.

This study hypothesized that the myocardial cell apoptosis caused by persistent ERS response was involved in the cardiac remodeling of heart failure, and berberine could reduce the myocardial cell apoptosis and improve cardiac remodeling through inhibiting ERS response, thereby improving the cardiac function. The rat model of heart failure after myocardial infarction was established, followed by berberine intervention, so as to study its effects on ERS-mediated myocardial cell apoptosis and its correlation with cardiac function, thereby investigating the mechanism of berberine improving the cardiac function of heart failure rats.

\section{Materials and methods}

Experimental animal and myocardial infarction model. Fifty female Wistar rats weighing 200-220 g were fed in the animal center for one week to adapt to the environment. After fasting for 4-6 h, under anesthesia via inhalation of ether, the rats were fixed on the operating table in a supine position; the chest hair was shaved and disinfected; and a purse was made in the chest to be opened using a no. 10 suture, and the pericardium was quickly opened to expose the heart. The coronary artery was found in the pulmonary arterial cone and left atrium; then the anterior descending coronary artery was ligatured using a no. 0 suture and the heart was placed back to the chest; the air and blood in the chest were squeezed out, and the purse and chest were quickly closed. The thoracotomy lasted for $<30 \mathrm{sec}$ in total. The steps for the Sham group were the same as those in the model group, except for stringing and no ligation. After operation, the rats received intraperitoneal injection of penicillin $(200,000 \mathrm{U} / \mathrm{kg})$ for 7 days to prevent infection. This study was approved by the Animal Ethics Committee of Fujian Medical University Animal Center (Fujian, China).

Grouping and administration. At 4 weeks after the myocardial infarction model was established, the rats were randomly divided into three groups: Sham-operation group (Sham group), myocardial infarction control group (Sal group) and berberine treatment group (Ber group). The control group and experimental group received the gavage with the same amount of normal saline and berberine $(20 \mathrm{mg} / \mathrm{kg})$ for 4 weeks.

Hemodynamic detection. After administration, rats received the intraperitoneal injection of $3 \%$ pentobarbital sodium for anesthesia, and left ventricular intubation via the right common carotid artery. The pressure transducer was connected to the carrier amplifier, the left ventricular end-systolic pressure (LVSP) and left ventricular end-diastolic pressure (LVEDP) were recorded using the 8-channel physiological recorder; the electric signal was input to the differentiator to trace the maximum ascending velocity of left ventricular pressure $(+\mathrm{dp} / \mathrm{dt})$ and the maximum descending velocity of left ventricular pressure (-dp/dt).

Determination of plasma BNP level. Before the treatment of rats, $0.5 \mathrm{ml}$ serum was taken from the rat, and $0.5 \mathrm{ml}$ tissue lysis buffer was added, followed by tissue homogenate at $4^{\circ} \mathrm{C}$ and centrifugation at $4^{\circ} \mathrm{C}$ for $10 \mathrm{~min}(1,750 \mathrm{x} \mathrm{g})$; the supernatant was taken and centrifuged again at $4^{\circ} \mathrm{C}$ for $30 \mathrm{~min}$ $(10,500 \times \mathrm{g})$, and then recycled. The specific procedures were carried out according to the instructions of the kit.

Pathomorphological detection of myocardial tissue. The pathomorphological changes in myocardium were evaluated via $H \& E$ staining. The pathomorphological changes in myocardial tissue were observed via an optical microscope (BX-42, Olympus Corporation, Tokyo, Japan). The degree of myocardial fibrosis was analyzed via Masson's staining: pale blue for collagen fibers, pink for muscle fibers, red blood cells and cytoplasm, and blue brown for nuclei.

Detection of apoptotic cells. TUNEL staining was applied to myocardial tissues, followed by counting the number of apoptotic cells under an optical microscope and then images were captured.

Immunohistochemistry. The expression levels of Bcl-2, Bax and caspase-3 were detected by immunohistochemistry. After the slices were treated according to the standard procedure, Bcl-2, Bax and caspase-3 primary antibody diluents were added for reaction at room temperature for $3 \mathrm{~h}$. Then the secondary antibody was used for $30 \mathrm{~min}$ incubation, followed by color development using the hypersensitivity kit according to the supplier's protocol. Rabbit polyclonal Bcl-2 antibody (1:100; cat. no. ab59348), rabbit monoclonal Bax antibody (1:100; cat. no. ab32503), rabbit polyclonal caspase-3 antibody (1:100; cat. no. ab13847) and secondary goat anti-rabbit (HRP) IgG antibody (1:1,000; cat. no. ab6721) were all purchased from Abcam (Cambridge, MA, USA).

Protein extraction and western blot analysis. The heart was taken from the rat and the total protein was extracted according to the standard procedure, followed by protein quantification via BCA method. Endoplasmic reticulum stress-related proteins were detected by western blot analysis. The concentration of separation gel (15\%) and the size of electrophoresis voltage were adjusted according to the molecular weight of target protein, followed by color development via chemiluminesence kit (Merck Millipore, Burlington, MA, USA) and 

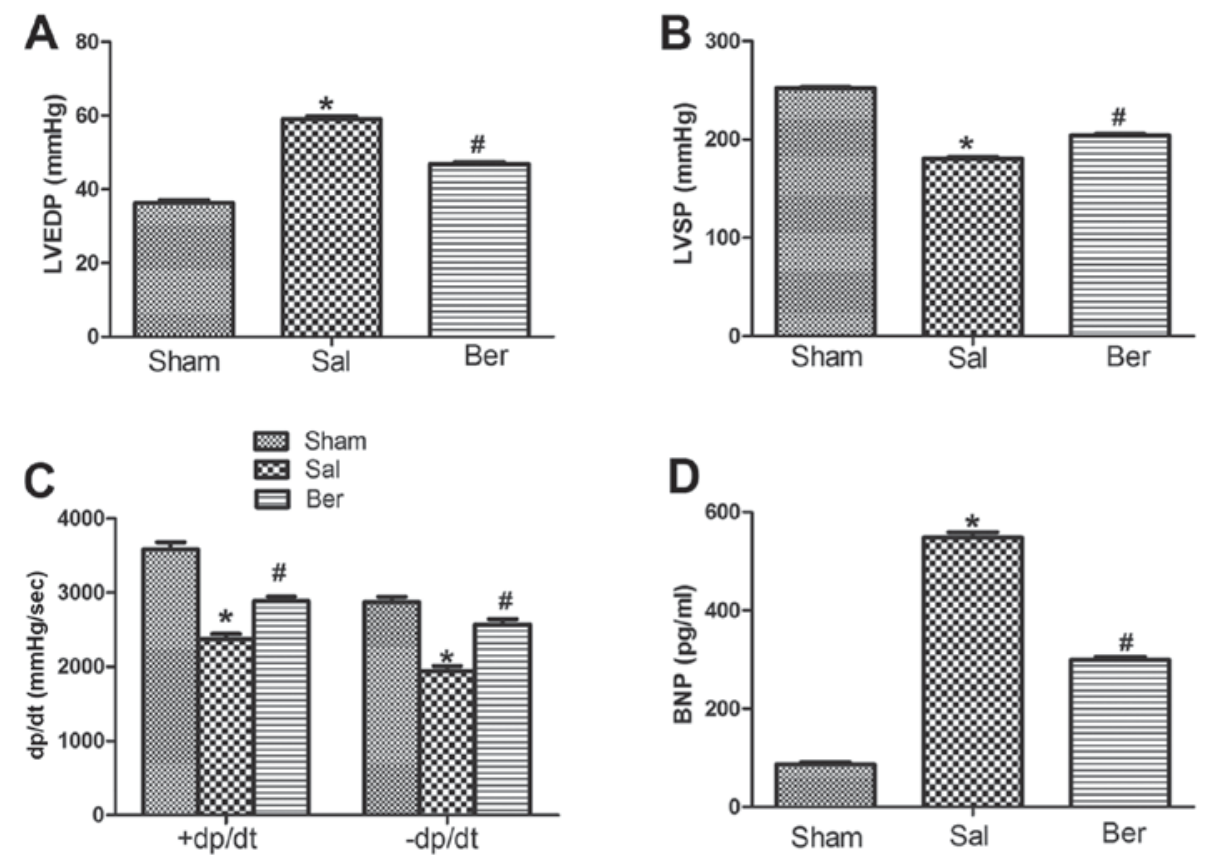

Figure 1. Berberine improves the cardiac function of rats after myocardial infarction. (A) Analysis of left ventricular end-diastolic pressure (LVEDP). (B) Analysis of left ventricular end-systolic pressure (LVSP). (C) Analysis of \pm dp/dt. (D) Analysis of BNP. ${ }^{*} \mathrm{P}<0.05$ vs. Sham group; ${ }^{*} \mathrm{P}<0.05$ vs. Sal group.

semi-quantitative analysis via gray value. Primary rabbit polyclonal GRP78 antibody (1:500; cat. no. ab21685); rabbit monoclonal CHOP antibody (1:500; cat. no. ab179823); rabbit polyclonal caspase-12 antibody (1:500; cat. no. ab62484); rabbit polyclonal GAPDH antibody (1:500, cat. no. ab37168) and secondary goat anti-rabbit (HRP) IgG antibody (1:2,000; cat. no.. ab6721) purchased from Abcam (Cambridge, MA, USA), were used for Western blot analysis.

RNA extraction and RT-PCR. According to the standard procedure, RNA in myocardial tissues was extracted, and the primer design and synthesis were completed by Shanghai Sangon Co. (Shanghai, China). Total RNA was taken to synthesize complementary deoxyribonucleic acid (cDNA) using the RT Revert Aid First Strand cDNA Synthesis kit (Thermo Fisher Scientific, Inc., Waltham, MA, USA). PCR was performed after reverse transcription according to the instructions of Invitrogen kit (Invitrogen, Carlsbad, CA, USA). ImageJ software (version X; Media Cybernetics, Silver Spring, MD, USA) was employed for determination of bands grey values. Primer sequences used in this study was according to previous reports $(11,12)$.

Statistical analysis. Data were analyzed using SPSS 17.0 statistical software (Chicago, IL, USA). Student's t-test was used for the comparison between the two groups, and one-way analysis of variance followed by post hoc test (Least Significant Difference) was used for the comparison of correlation among groups. $\mathrm{P}<0.05$ suggested that the difference was statistically significant.

\section{Results}

Berberine improves the cardiac function of rats after myocardial infarction. Compared with those in Sham group,
LVEDP in Sal group was significantly increased, but LVSP and $\pm \mathrm{dp} / \mathrm{dt}$ were significantly decreased at 8 weeks after myocardial infarction. After berberine treatment, LVEDP was decreased and LVSP and $\pm \mathrm{dp} / \mathrm{dt}$ were increased. At the end of experiment, plasma BNP levels in the Sham, Sal and Ber groups were measured. Compared with that in Sham group, BNP level in Sal group was significantly increased. By contrast, it was found that berberine significantly decreased the BNP level (Fig. 1).

Berberine improves the myocardial fibrosis after myocardial infarction. H\&E staining showed that the myocardial cells in Sham group were neatly arranged with clear transverse striations. The myocardial cells in Sal group were swollen with irregular shape and disordered arrangement, and the gap of myocardial cells was filled with a large number of fibrous tissues, and the transverse striations were blurry and broken. After berberine treatment, myocardial cells were neatly arranged with decreased degree of fibroplasias (Fig. 2A). Masson's staining showed that myocardial cells dominated in the Sham group, and there were no obvious myocardial interstitial and collagen components. In Sal group, there were obvious collagen components with a small number of myocardial cells. After berberine treatment, the number of myocardial cells and nuclei was significantly increased, but the collagen component was significantly decreased (Fig. 2B).

Berberine reduces cell apoptosis after myocardial infarction. TUNEL staining showed that compared with that in Sham group, the myocardial cell apoptosis in Sal group was significantly increased, suggesting that berberine can inhibit myocardial cell apoptosis of heart failure (Fig. 3A). Immunohistochemical detection showed yellow brown staining region in the three groups of myocardial tissues, indicating that the protein expression in $\mathrm{Bcl}-2$, Bax and 

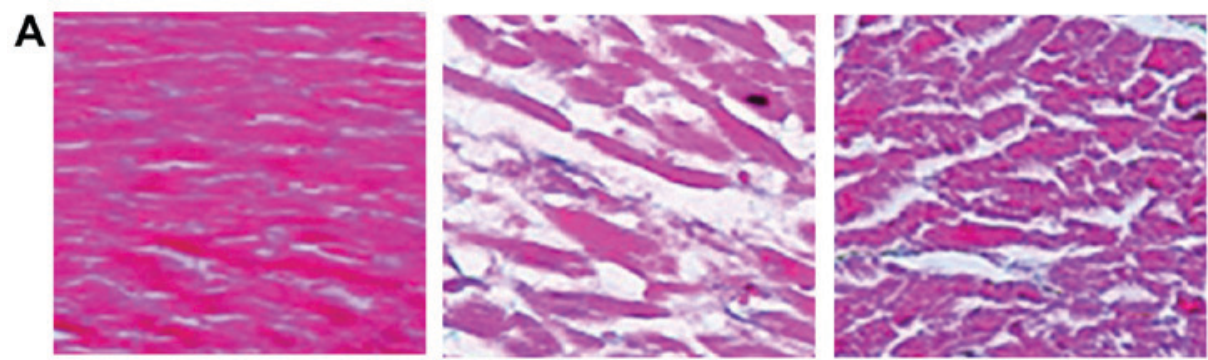

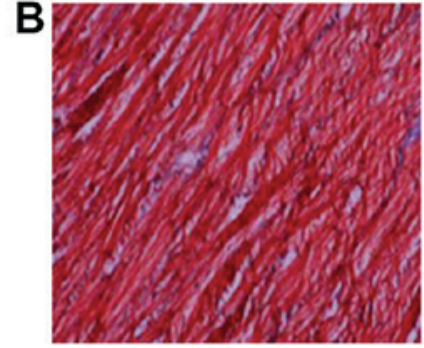

Sham

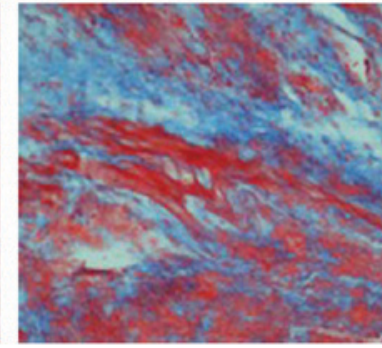

Sal

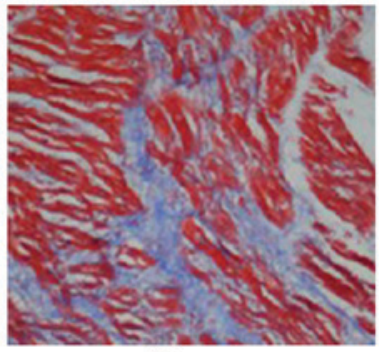

Ber

Figure 2. Berberine improves myocardial fibrosis after myocardial infarction. (A) Representative H\&E staining images of the peri-infarct area of rats. (B) Representative Masson's staining images of the peri-infarct area of rats.
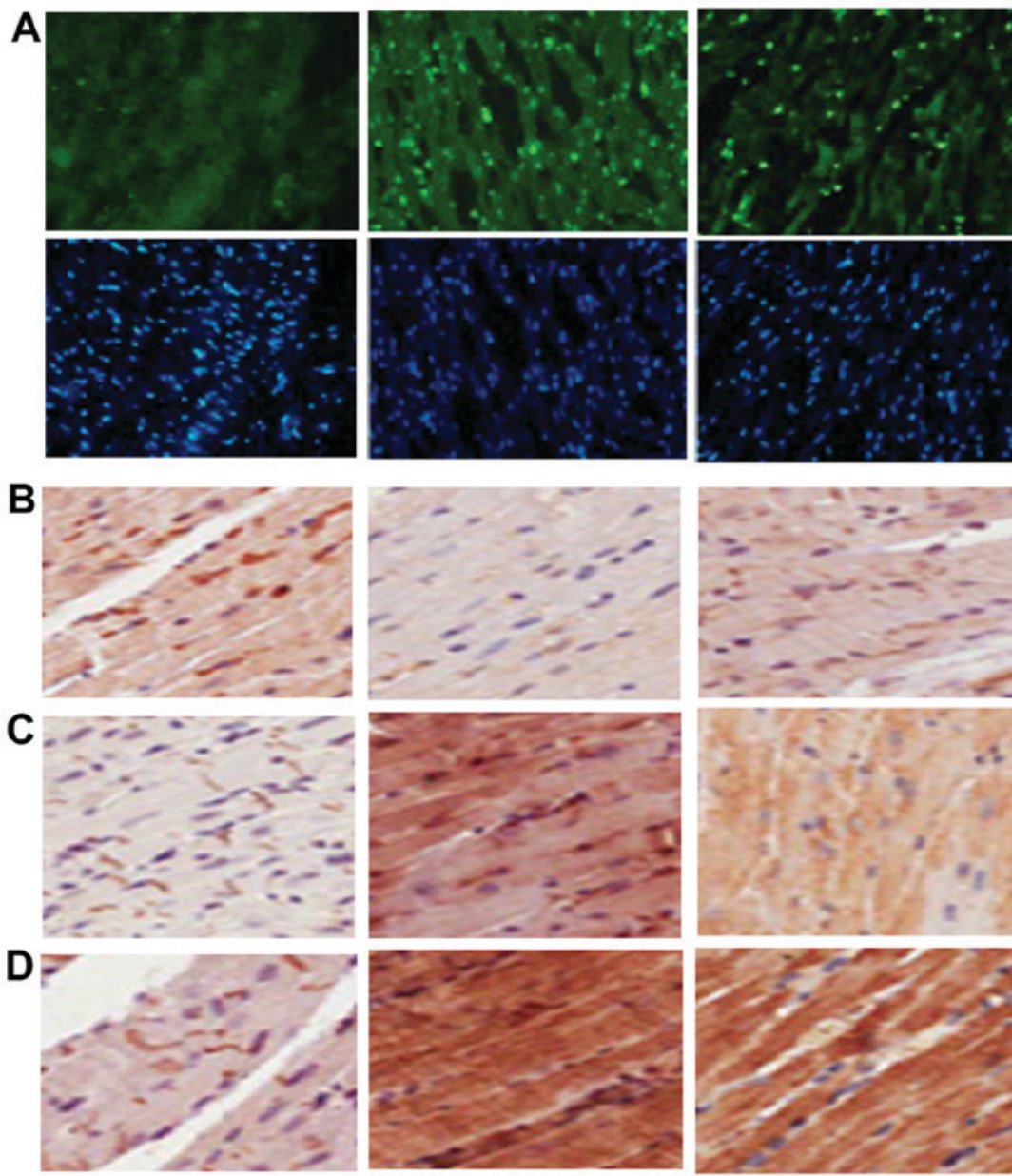

TUNEL

DAPI

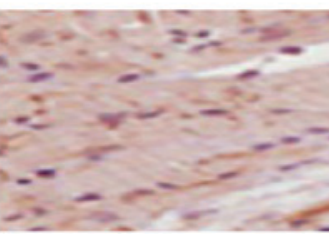

Bcl-2
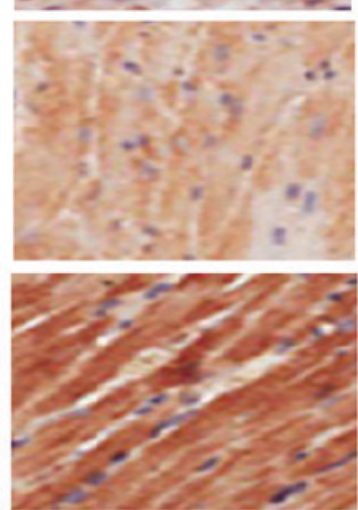

Bax

\section{Caspase-3}

Figure 3. Berberine reduces cell apoptosis after myocardial infarction. (A) Representative TUNEL images of the peri-infarct area of rats. Representative immunohistochemical images of (B) Bcl-2, (C) Bax, and (D) caspase-3.

caspase- 3 existed in the three groups of myocardial tissues. Compared with those in Sham group, Bcl-2 in Sal group was significantly decreased, but Bax and caspase- 3 were significantly increased, suggesting that berberine can increase the 
A
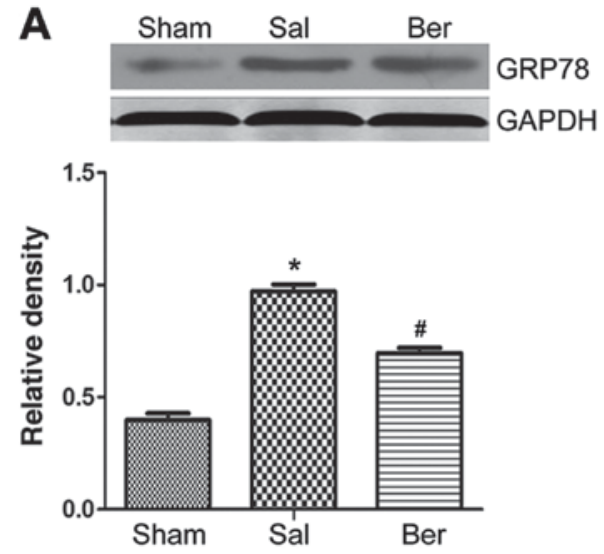

C
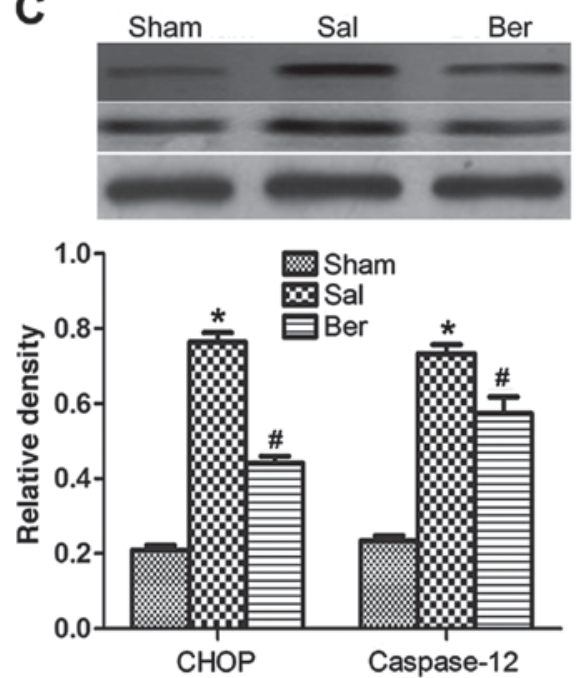

B

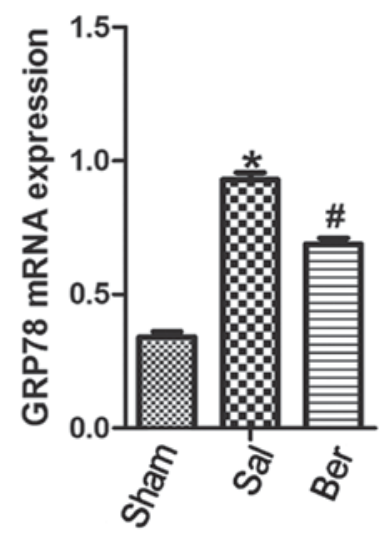

D

\section{CHOP \\ Caspase-12 GAPDH}

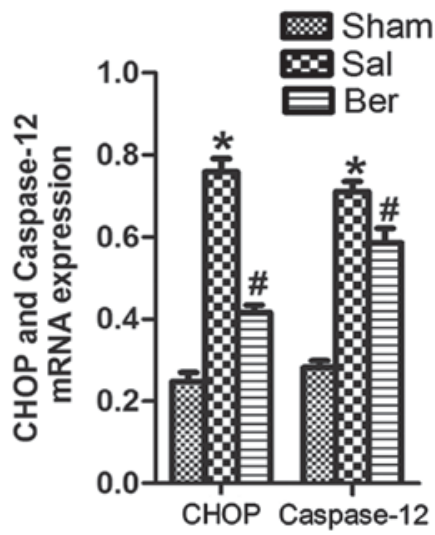

Figure 4. Berberine alleviates endoplasmic reticulum stress (ERS) response after myocardial infarction. (A) Western blot analyses reveals the expression of GRP78. (B) RT-PCR analysis reveals the level of GRP78 mRNA. (C) Western blot analyses reveals the expression of CHOP and caspase-12. (D) RT-PCR analysis reveals the level of CHOP and caspase-12 mRNA. ${ }^{*} \mathrm{P}<0.05$ vs. Sham group; ${ }^{\#} \mathrm{P}<0.05$ vs. Sal group.

expression of Bcl-2 and decrease the expression of Bax and caspase-3 (Fig. 3B-D).

Berberine alleviates ERS response after myocardial infarction. The results of western blot analysis showed that the expression of GRP78 (Fig. 4A and B), CHOP and caspase-12 (Fig. 4C and D) in myocardium was increased significantly after myocardial infarction, suggesting that berberine can significantly decrease the expressions of GRP78, CHOP and caspase-12. The results of RT-PCR further proved that the mRNA expression in GRP78, CHOP and caspase-12 were increased after myocardial infarction, suggesting that berberine can significantly reduce the mRNA expression in GRP78, CHOP and caspase-12 (Fig. 4).

\section{Discussion}

Myocardial infarction leads to heart failure and to changes in cardiac hemodynamics. LVEDP is an index reflecting the left ventricular preload, which depends on the returned blood volume before ventricular systole and cardiac ejection function. The maximum ascending velocity $(+\mathrm{dp} / \mathrm{dt})$ of left ventricular pressure during isovolumetric contraction phase reflects the maximum velocity of tension changes in ventricular wall in systole. It is affected by cardiac afterload and myocardial contractility, which is an important index to evaluate myocardial contractility. The maximum descending velocity $(-\mathrm{dp} / \mathrm{dt})$ of left ventricular pressure during isovolumetric relaxation phase is mainly affected by the preload of myocardial relaxation. $\pm \mathrm{dp} / \mathrm{dt}$ will be decreased and LVEDP will be increased when myocardial systolic and diastolic function is inhibited (18). In this experiment, compared with that in Sham group, LVEDP in Sal group was significantly increased and $\pm \mathrm{dp} / \mathrm{dt}$ was decreased, indicating that the myocardial systolic and diastolic function of rats with heart failure after myocardial infarction was impaired. Berberine can reduce LVEDP, improve LVSP and $\pm \mathrm{dp} / \mathrm{dt}$ and decrease the plasma BNP level, indicating that berberine can improve the cardiac function of heart failure after myocardial infarction.

Cardiac remodeling occurs after myocardial infarction, which is manifested as the disordered arrangement of myocardial cells and significant increase of myocardial fibrosis in heart morphology (19). In this study, myocardial cells were arranged disorderly and myocardial fibrosis was obvious with cardiac remodeling in non-infarcted zone in Sal group. Berberine can 
significantly inhibit the left ventricular myocardial fibrosis of heart failure and reduce cardiac remodeling.

Previous findings showed that, at 1-6 h after myocardial infarction, the frequency of myocardial cell apoptosis away from the infarcted zone was significantly correlated with LVEDP, reflecting the severity of left ventricular insufficiency (20). In addition, the nitric oxide synthase gene deletion via gene knockout showed that the number of apoptotic myocardial cells was decreased and LVEF was increased after myocardial infarction, suggesting that the improvement of cardiac function in heart failure is closely related to the decrease of frequency of myocardial cell apoptosis away from the infarcted zone, and the myocardial cell apoptosis away from the infarcted zone may be one of the important reasons for cardiac remodeling and development of heart failure after myocardial infarction (20). In heart failure, the hypoxia-ischemia of myocardial cells, oxidative stress, inflammatory factors, mechanical load and neuroendocrine factors, can induce myocardial cell apoptosis $(21,22)$, but which factor dominates in heart failure remains unclear, and the specific mechanism of myocardial apoptosis and relationship need to be further studied. At present, animal experiments and autopsy of patients with heart failure have shown that myocardial cell apoptosis exists in heart failure, but the proportion of apoptosis varies in different studies. TUNEL staining is a sensitive index of evaluating apoptosis. In this study, the level of myocardial cell apoptosis was evaluated by TUNEL staining. It was found that the proportion of myocardial cell apoptosis in non-infarcted zone in heart failure after myocardial infarction was much higher than that in Sham group, and such a change was related to the severity of cardiac remodeling; and the increased level of myocardial cell apoptosis in Sal group was related to the increased levels of plasma BNP and LVEDP and the decreased levels of LVSP and $\pm \mathrm{dp} / \mathrm{dt}$, which was consistent with the results in most literature, suggesting that myocardial cell apoptosis is involved in the cardiac remodeling in heart failure after myocardial infarction, deteriorating the cardiac function.

Bcl-2 and Bax are the most important members of Bcl-2 family, which are involved in the regulation of apoptosis (23): The decreased protein expression of $\mathrm{Bcl}-2$, the increased protein expression of Bax and the downregulation of $\mathrm{Bcl}-2 / \mathrm{Bax}$ protein expression level induce cell apoptosis. In this study, it was found that $\mathrm{Bcl}-2$ protein expression was decreased in heart failure after myocardial infarction, and Bax protein expression was increased, which was consistent with increased myocardial cell apoptosis. After berberine treatment, Bcl-2 protein expression was increased, Bax protein expression was decreased and the apoptosis level was decreased, suggesting that berberine inhibits the myocardial cell apoptosis by upregulating the expression of $\mathrm{Bcl}-2 / \mathrm{Bax}$. Caspase-3 is a member of caspase family, which is an important downstream effector molecule in the apoptosis process and the executor of apoptosis (24). The results of this experiment showed that caspase- 3 protein expression in myocardial tissue of heart failure after myocardial infarction was increased, but caspase-3 protein expression was decreased after berberine intervention, suggesting that the inhibitory effect of berberine on caspase-3 expression is involved in the regulation of apoptosis, thus promoting cell survival.

Previous findings have confirmed ERS and ERS-induced myocardial cell apoptosis existed in the pathological process of heart failure, which is manifested as the increased GRP78 expression $(25,26)$ of ERS response and the activation of ERS-mediated CHOP and caspase-12 pathways $(12,27)$. The rat model of heart failure after myocardial infarction was established in this experiment. WB detection showed that compared with that in Sham group, GRP78 protein expression was increased; RT-PCR showed mRNA expression in GRP78 was increased, indicating that ERS response is activated in heart failure after myocardial infarction, and protein and mRNA expression of CHOP and caspase-12 are increased. TUNEL staining showed that the number of apoptotic myocardial cells in heart failure was increased, indicating that ERS response promotes myocardial cell apoptosis through the above apoptotic signaling pathways, which is consistent with the result of previous studies. The protein and mRNA expression of GRP78, CHOP and caspase-12 were downregulated by berberine, and the ratio of positive cells in TUNEL staining was decreased compared with that in Sal group, indicating that berberine inhibits the myocardial cell apoptosis through inhibiting the ERS response in heart failure after myocardial infarction and ERS-induced CHOP and caspase-12 apoptosis signaling pathways.

In conclusion, berberine can inhibit the myocardium cell apoptosis of heart failure after myocardial infarction, and its mechanism may be realized through affecting the ERS in myocardial tissue of heart failure after myocardial infarction and CHOP and caspase-12 apoptotic signaling pathway, upregulating Bcl-2/Bax expression and downregulating caspase-3 expression, thus inhibiting cardiac remodeling and protecting the cardiac function.

\section{Acknowledgements}

Not applicable.

\section{Funding}

This study was supported by the Medical Innovation Project of Fujian Provice of China (2014-XB-30) and the Natural Science Foundation of Fujian Provice of China (2018J01406).

\section{Availability of data and materials}

The datasets used and/or analyzed during the present study are available from the corresponding author on reasonable request.

\section{Authors' contributions}

YL, KC and XD conceived and designed the study. WL, GH and GL were responsible for the analysis of the animal data. WS, LC and YF interpreted the western blot and PCR data and drafted the manuscript. YL revised the manuscript critically for important intellectual content. All authors read and approved the final manuscript.

\section{Ethics approval and consent to participate}

This study was approved by the Animal Ethics Committee of Fujian Medical University Animal Center (Fujian, China). 


\section{Patient consent for publication}

Not applicable.

\section{Competing interests}

The authors declare that they have no competing interests.

\section{References}

1. Deng $\mathrm{J}$ and Zhong Q: Advanced research on the microRNA mechanism in heart failure. Int J Cardiol 220: 61-64, 2016.

2. Liu L, Wu J and Kennedy DJ: Regulation of cardiac remodeling by cardiac $\mathrm{Na}(+) / \mathrm{K}(+)-A T P a s e$ isoforms. Front Physiol 7: 382, 2016.

3. Karaye KM, Lindmark K and Henein MY: One year survival in nigerians with peripartum cardiomyopathy. Heart Views 17 55-61, 2016.

4. Sung MM and Dyck JR: Therapeutic potential of resveratrol in heart failure. Ann NY Acad Sci 1348: 32-45, 2015.

5. Zhao Z, Luo J, Ma L, Luo X and Huang L: Effect of granulocyte colony stimulating EPC on cardiac function and myocardial energy expenditure in patients with heart failure after myocardial infarction. Int J Clin Exp Med 8: 16578-16584, 2015.

6. Bonnefoy E and Kirkorian G: Mortality of myocardial infarction. Ann Cardiol Angeiol (Paris) 60: 311-316, 2011 (In French).

7. Roig T, Márquez MA, Hernández E, Pineda I, Sabartés O, Miralles R and Inzitari M: Geriatric assessment and factors associated with mortality in elderly patients with heart failure admitted to an acute geriatric unit. Rev Esp Geriatr Gerontol 48: 254-258, 2013 (In Spanish).

8. Alpert MA, Omran J and Bostick BP: Effects of obesity on cardiovascular hemodynamics, cardiac morphology, and ventricular function. Curr Obes Rep 5: 424-434, 2016.

9. Suarez G and Meyerrose G: Heart failure and galectin 3. Ann Transl Med 2: 86, 2014.

10. Kinoshita T, Ishikawa Y, Arita M, Akishima-Fukasawa Y, Fujita K, Inomata N,Suzuki T, Namiki A, Mikami T, Ikeda T, et al: Antifibrotic response of cardiac fibroblasts in hypertensive hearts through enhanced TIMP-1 expression by basic fibroblast growth factor. Cardiovasc Pathol 23: 92-100, 2014.

11. Park CS, Cha H, Kwon EJ, Sreenivasaiah PK and Kim DH: The chemical chaperone 4-phenylbutyric acid attenuates pressureoverload cardiac hypertrophy by alleviating endoplasmic reticulum stress. Biochem Biophys Res Commun 421: 578-584, 2012.

12. Xin W, Li X, Lu X, Niu K and Cai J: Involvement of endoplasmic reticulum stress-associated apoptosis in a heart failure model induced by chronic myocardial ischemia. Int J Mol Med 27: 503-509, 2011.

13. Chen K, Li G, Geng F, Zhang Z, Li J, Yang M, Dong L and Gao F: Berberine reduces ischemia/reperfusion-induced myocardial apoptosis via activating AMPK and PI3K-Akt signaling in diabetic rats. Apoptosis 19: 946-957, 2014.

14. Chang W, Li K, Guan F, Yao F, Yu Y, Zhang M, Hatch GM and Chen L: Berberine pretreatment confers cardioprotection against ischemia-reperfusion injury in a rat model of type 2 diabetes. J Cardiovasc Pharmacol Ther 21: 486-494, 2016.
15. Doggrell SA: Berberine - a novel approach to cholesterol lowering. Expert Opin Investig Drugs 14: 683-685, 2005.

16. Liu JC, Chan P, Chen YJ, Tomlinson B, Hong SH and Cheng JT: The antihypertensive effect of the berberine derivative 6-protoberberine in spontaneously hypertensive rats. Pharmacology 59 283-289, 1999 .

17. Imenshahidi $M$ and Hosseinzadeh $H$ : Berberis vulgaris and berberine: An update review. Phytother Res 30: 1745-1764, 2016.

18. Seara FAC, Maciel L, Barbosa RAQ, Rodrigues NC, Silveira ALB, Marassi MP, Carvalho AB, Nascimento JHM and Olivares EL: Cardiac ischemia/reperfusion injury is inversely affected by thyroid hormones excess or deficiency in male Wistar rats. PLOS ONE 13: e190355, 2018.

19. Meijers WC, van der Velde AR, Pascual-Figal DA and de Boer RA: Galectin-3 and post-myocardial infarction cardiac remodeling. Eur J Pharmacol 763: 115-121, 2015.

20. Sam F, Sawyer DB, Chang DL, Eberli FR, Ngoy S, Jain M, Amin J, Apstein CS and Colucci WS: Progressive left ventricular remodeling and apoptosis late after myocardial infarction in mouse heart. Am J Physiol Heart Circ Physiol 279: H422-H428, 2000.

21. Li S, Feng J, Wei S, Qian X, Cao J and Chen B: Delayed neutrophil apoptosis mediates intermittent hypoxia-induced progressive heart failure in pressure-overloaded rats. Sleep Breath 20: 95-102, 2016.

22. Barajas-Espinosa A, Basye A, Angelos MG and Chen CA: Modulation of p38 kinase by DUSP4 is important in regulating cardiovascular function under oxidative stress. Free Radic Biol Med 89: 170-181, 2015.

23. Zhang W, Shi HY and Zhang M: Maspin overexpression modulates tumor cell apoptosis through the regulation of Bcl-2 family proteins. BMC Cancer 5: 50, 2005.

24. Sun Y, Lin Y,Li H, Liu J, Sheng X and Zhang W: 2,5-Hexanedione induces human ovarian granulosa cell apoptosis through BCL-2, BAX, and CASPASE-3 signaling pathways. Arch Toxicol 86: 205-215, 2012.

25. Gupta MK, Tahrir FG, Knezevic T, White MK, Gordon J, Cheung JY, Khalili K and Feldman AM: GRP78 interacting partner Bag5 responds to ER stress and protects cardiomyocytes from ER stress-induced apoptosis. J Cell Biochem 117: 1813-1821, 2016.

26. Liu L, Wang HJ, Xin Q, Zhou XM, Zhao YJ, Huang X and Zhao M: The potential effects of endoplasmic reticulum stress on the apoptosis of myocardial cells from mice with heart failure induced by acute viral myocarditis caused by B3 Coxsackie virus. Zhongguo Ying Yong Sheng Li Xue Za Zhi 30: 461-464, 2014 (In Chinese).

27. Fu HY, Minamino T, Tsukamoto O, Sawada T, Asai M, Kato H, Asano Y, Fujita M, Takashima S, Hori M, et al: Overexpression of endoplasmic reticulum-resident chaperone attenuates cardiomyocyte death induced by proteasome inhibition. Cardiovasc Res 79: 600-610, 2008.

(7)(9) This work is licensed under a Creative Commons Attribution-NonCommercial-NoDerivatives 4.0 International (CC BY-NC-ND 4.0) License. 